\title{
Effect of transgenic human insulin-like growth factor-1 on spinal motor neurons following peripheral nerve injury
}

\author{
JIAXIANG GU* , HONGJUN LIU* , NAICHEN ZHANG, HENG TIAN, \\ JUNBO PAN, WENZHONG ZHANG and JINGCHENG WANG
}

Department of Hand Surgery, Clinical Medical Institute of Yangzhou University, Yangzhou, Jiangsu 225001, P.R. China

Received September 24, 2013; Accepted February 3, 2015

DOI: 10.3892/etm.2015.2472

\begin{abstract}
The aim of the present study was to observe the protective effect of exogenous human insulin-like growth factor-1 (hIGF-1) on spinal motor neurons, following its local transfection into an area of peripheral nerve injury. A total of 90 male Wistar rats that had been established as sciatic nerve crush injury models were randomly divided into three groups: hIGF-1 treatment, sham-transfected control and blank control groups. The different phases of hIGF-1 expression were observed in the spinal cord via postoperative immunostaining and the apoptosis of motor neurons was observed using the terminal deoxynucleotidyl transferase-mediated dUTP nick end labeling method. Pathological changes of the motor neurons and Nissl bodies within cell bodies were observed via Marsland and Luxol fast blue double staining, while changes in the neuropil of the spinal cord anterior horn were investigated via ultrastructural observation. It was found that hIGF-1, locally transfected into an area of peripheral nerve injury, was expressed in the spinal anterior horn following axoplasmic transport; the peak hIGF-1 expression occurred approximately a week following transfection. The number of apoptotic spinal cord motor neurons observed in the hIGF-1 treatment group was fewer than that in the sham-transfected and blank control groups at days 7, 14 and 21 following transfection $(\mathrm{P}<0.01)$. Furthermore, the quantity of motor neuron cells in the anterior horn of the spinal cord in the hIGF-1 treatment group was higher compared with those in the sham-transfected and blank control groups at days 2, 7, 14 and 28 following transfection $(\mathrm{P}<0.01)$. The degenerative changes of Nissl bodies within the cytoplasm of the hIGF-1 treatment group were less severe compared with those of the sham-transfected and blank control
\end{abstract}

Correspondence to: Dr Jingcheng Wang, Department of Hand Surgery, Clinical Medical Institute of Yangzhou University, 98 Nantong West Road, Yangzhou, Jiangsu 225001, P.R. China E-mail: jingwangcn@163.com

\section{${ }^{*}$ Contributed equally}

Key words: peripheral nerve, human insulin-like growth factor-1, spinal cord, neuropil groups. At day 56 following transfection, the spinal anterior horn neuropil ultrastructure in the hIGF-1 treatment group was generally normal, while the sham-transfected and blank control groups exhibited an increased number of protruding gaps and local cavities. These results indicate that the application of exogenous hIGF-1 is capable of protecting spinal cord motor neurons following peripheral nerve injury.

\section{Introduction}

Peripheral nerve injury leads to the damage of axons, where neurons lose the basis of survival and gradually degenerate or disappear as a result of the interruption of axonal transport. Regeneration of the injured peripheral nerve is dependent on the vitality of the central neurons and the nerve regeneration microenvironment, while the death of neurons exacerbates the peripheral nerve injury (1). Therefore, the key to preventing or reducing the occurrence of degenerative cell death may be the protection of the motor neuron cell body following peripheral nerve injury. Thus, it is necessary to identify methods of protecting motor neurons and to develop a novel repair technique.

Insulin-like growth factor-1 (IGF-1), identified more than 30 years ago as a type of biologically active peptide with a role in the central and peripheral nervous systems, exhibits neurotrophic and neural regulatory effects (2). Studies on IGF-1 have predominantly focused on diseases such as diabetes, osteoporosis and Alzheimer's disease (3-5). A number of animal studies have investigated the role of IGF-1 in the treatment of peripheral nerve injuries (6-8), including injuries involving the facial nerves and sciatic nerve. However, studies investigating the effects of IGF-1 on the pathological changes of spinal motor neurons following peripheral nerve injury are less common. As the half-life of IGF-1 is short and repeated transfusions are required, genetic engineering may be performed to solve the problem of long-term IGF-1 supply. In the present study, IGF-1 was applied to the area of sciatic nerve injury in order to observe the protective effects on spinal cord neurons.

With advances in basic research, cell transplantation, tissue engineering technology and genetic engineering technology, the analysis of peripheral nerve repair has been enriched and developed. Numerous nutritional factors have been found to be associated with nerve regeneration, including brain-derived 
neurotrophic factor (9), glial cell line-derived neurotrophic factor (10), ciliary neurotrophic factor (11) and IGF-1. IGF-1, a multifunctional biological peptide that has a role in the central and peripheral nervous systems, exhibits neurotrophic and neural regulatory effects. At present, the majority of studies have focused on the central nervous system (12), while there is limited literature regarding the promotion of peripheral nerve regeneration by IGF-1 following peripheral nerve injury and its effect on the pathological changes of spinal motor neurons. The present study investigated the protective effects of IGF-1 on spinal motor neurons following peripheral nerve injury by applying transgenic IGF-1 to a sciatic nerve injury site.

According to the hypothesis of nerve regeneration in small gaps (13), with the appropriate clearance, neural nutrition and chemotaxis can be used in nerve regeneration to solve the phenomenon of dislocated nerve growth and to achieve more accurate docking $(14,15)$. In the present study, a rat model of sciatic nerve crush injury was developed and plasmid-liposome-mediated human IGF-1 (hIGF-1) was introduced into the nerve regeneration chamber via epineural injection. The protective effects of hIGF-1 on the spinal motor neurons at various time points following peripheral nerve injury were then observed.

Through the analysis of hIGF-1 expression, apoptosis detection and transmission electron microscopy (TEM) observations of motor neurons in the anterior horn of the spinal cord, the protective effect of internal transgenic hIGF-1 on spinal motor neurons following peripheral nerve injury was investigated, as well as the underlying mechanism.

\section{Materials and methods}

Experimental animals and reagents. A total of 90 adult male Wistar rats, weighing 200-250 g, were provided by the Experimental Animal Center of Jilin University (Changchun, China). Positive liposomes (Lipofectamine ${ }^{\circledR} ; 2 \mu \mathrm{g} / \mu \mathrm{l}$ ) and pcDNA3.1 $(1 \mu \mathrm{g} / \mu \mathrm{l})$ were purchased from Beijing Yuanping Hao Biotechnology Ltd (Beijing, China) and pcDNA-hIGF-1 $(1 \mu \mathrm{g} / \mu \mathrm{l})$ was supplied by Dr Bo Shen from the Public Health School of Jilin University (Changchun, China). Liposomes and plasmids were mixed at a mass ratio of 1.5:1. The mouse anti-human IGF-1 polyclonal antibody (\#MS-1508; PcAb) and streptavidin-peroxidase (SP) kit were purchased from the Fujian Maixin Biotechnology Development Co. (Fuzhou, China) and the terminal deoxynucleotidyl transferase-mediated dUTP nick end labeling (TUNEL) kit was purchased from Wuhan Boster Biological Technology, Ltd. (Wuhan, China). The current study was approved by the Institutional Animal Care and Use Committee of the Experimental Animal Center of Jilin University.

Establishing animal models and groupings. The rats were randomly divided into three groups: hIGF-1 treatment, sham-transfected control and blank control groups $(n=30$ per group). Ketamine hydrochloride $(5 \% ; 130 \mathrm{mg} / \mathrm{kg}$ ) was injected intraperitoneally to anesthetize the rats and the right sciatic nerve was exposed. The sciatic nerve $5 \mathrm{~mm}$ below the piriformis muscle was clamped three times using a clamp of $3 \mathrm{~mm}$ width, and squeezed for $10 \mathrm{sec}$ each time, with observation conducted at a magnification of $x 6$ under an operating microscope. Next, $10 \mu \mathrm{l}$ pcDNA-hIGF-1 (hIGF-1 DNA, $4 \mu \mathrm{g}$ ) and Lipofectamine transfection reagent was immediately injected into the clamped epineurium of the rats in the hIGF-1 treatment group. The rats of the sham-transfected group were injected with a pcDNA3.1, Lipofectamine and distilled water mixture $(10 \mu \mathrm{l})$ and the blank control group was not injected with any substance. The animals were maintained in a single cage following surgery.

\section{Observation methods}

Immunohistochemical staining of hIGF-1 expression by motor neurons in the anterior horn of the spinal cord. Rats from each group were anaesthetized by the intraperitoneal injection of $5 \%$ ketamine hydrochloride and then perfused with $4 \%$ formaldehyde for whole animal fixation at days 2, 7, 14 and 28 postoperatively. The L4-6 spinal cord segments of the animals in each group were obtained and placed in $4 \%$ paraformaldehyde in a refrigerator at $4^{\circ} \mathrm{C}$ overnight. The samples were embedded in paraffin and IGF-1 expression levels in the L4-6 spinal cord segments were detected by incubation with a primary mouse anti-hIGF-1 PcAb (1:500) antibody overnight at $4^{\circ} \mathrm{C}$ followed by treatment with a goat anti-mouse polyclonal IgG secondary antibody (\#MS-642; 1:1,000; Fujian Maixin Biotechnology Development Co.) for $1 \mathrm{~h}$ at room temperature. The expression levels were evaluated using an image analysis system (HPIAS-1000; Shimadzu Corporation, Kyoto, Japan). In this system, the brown or brownish yellow positive staining was evaluated as a gray scale value. The SP kit reagent that provided the brown staining was anti-Mouse HIGF-1 supplied by $\sim 100 \mu \mathrm{g}$ antiserum lyophilized from a $0.2 \mu \mathrm{m}$ filtered solution in phosphate-buffered saline (PBS). These values were inversely related to the quantity of IGF-1 expressed. Three samples per group were analyzed at each time point.

Detection of apoptosis of motor neurons in the anterior horn of the spinal cord. Apoptosis was detected in L4-6 spinal cord motor neurons using the TUNEL detection kit, following polyformaldehyde perfusion at days 7, 14 and 21 after surgery. Three samples per group were analyzed at each time point.

Counting of motor neuron cells of the anterior horn in the spinal cord and observation of pathological changes. Spinal cord L4-6 paraffin-embedded sections, which were perfused with polyformaldehyde at days 2, 7, 14 and 28 following surgery, were double-stained with Marsland and Luxol fast blue. The form and number of spinal anterior horn cells, cytoplasmic Nissl bodies and the proliferation of glial cells were observed using a HPIAS-1000 Color pathological image analysis system (Shimadzu Corporation). Three samples per group were analyzed at each time point.

TEM observation. On day 56, L4-6 spinal cord anterior horns were obtained from five rats in each group and placed in $25 \mathrm{~g} / 1$ glutaraldehyde for prefixation and $10 \mathrm{~g} / 1 \mathrm{osmium}$ tetroxide for postfixation. The samples were then fixed in a series of ethanol dehydrates and epoxy-embedded in epon 812 (Haide Biotechnology Co., Ltd., Beijing, China). Ultra-thin sections were cut using an LKB Ultratome III (Pharmacia, Stockholm, Sweden) and then uranyl acetate and lead citrate double-staining was performed. Next, the ultrastructure of the neuropil within the spinal cord anterior horn was observed using radiography and a JEM-1200EX transmission electron microscope (Jeol, Tokyo, Japan). 
Table I. hIGF-1 protein expression levels in the spinal cord determined by immunohistochemistry $\left(\right.$ gray values $\left.^{\mathrm{a}}\right)$.

\begin{tabular}{lcccc}
\hline Group & Day 2 & Day 7 & Day 14 & Day 28 \\
\hline Blank control & $158.38 \pm 2.070$ & $154.23 \pm 2.900^{\mathrm{b}}$ & $154.52 \pm 2.200^{\mathrm{b}}$ & $161.70 \pm 2.030^{\mathrm{c}, \mathrm{d}}$ \\
Sham-transfected & $158.04 \pm 2.230$ & $153.59 \pm 2.610^{\mathrm{b}}$ & $156.13 \pm 2.110^{\mathrm{b}}$ & $160.98 \pm 2.400^{\mathrm{c}, \mathrm{d}}$ \\
hIGF-1 treatment & $143.87 \pm 2.851^{\mathrm{e}}$ & $133.39 \pm 2.111^{\mathrm{b}, \mathrm{e}, \mathrm{f}}$ & $135.41 \pm 2.351^{\mathrm{b}, \mathrm{e}, \mathrm{f}}$ & $143.69 \pm 2.451^{\mathrm{c}-\mathrm{f}}$ \\
\hline
\end{tabular}

Values are presented as the mean \pm standard deviation ( $\mathrm{n}=3$ per group per time point). ${ }^{\text {a }}$ The gray values are inversely correlated with the quantity of hIGF- 1 expressed. ${ }^{b} \mathrm{P}<0.01$ vs. day 2 ; ${ }^{\mathrm{c}} \mathrm{P}<0.01$ vs. day $7 ;{ }^{\mathrm{d}} \mathrm{P}<0.01$ vs. day $14 ;{ }^{\mathrm{e}} \mathrm{P}<0.01$ vs. the blank control group; ${ }^{\text {f }} \mathrm{P}<0.01$ vs. the sham-transfected group. hIGF-1, human insulin growth factor-1.

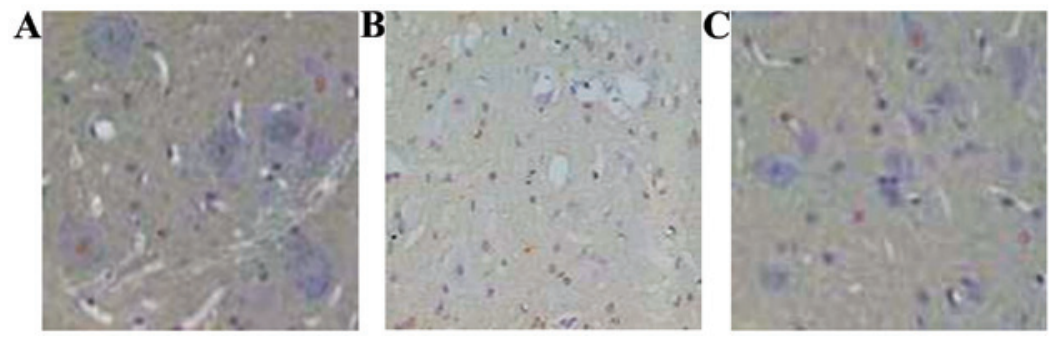

Figure 1. Detection of apoptosis by terminal deoxynucleotidyl transferase-mediated dUTP nick end labeling. Three weeks after treatment, the number of apoptotic cells in the (A) hIGF-1 treatment group was lower than in the (B) sham-transfected and (C) blank control groups (magnification, x100). The apoptotic cells were motor neuron cells and glial cells.

Statistical analysis. Results were statistically analyzed with SPSS statistical analysis software, version 11.5 (SPSS, Inc., Chicago, IL, USA), using multiple-sample mean variance analysis. Data are expressed as the mean \pm standard deviation. $\mathrm{P}<0.05$ was considered to indicate a statistically significant difference.

\section{Results}

hIGF-1 expression levels in the motor neurons in the anterior horn of the spinal cord

Comparison between time points for each group. The hIGF-1 expression levels in the motor neurons in the anterior horn of the spinal cord were compared on different days. A small amount of brown positive product was observed in the hIGF-1 treatment group at day 2 following the injection of exogenous hIGF-1 to treat the nerve injury. The quantity of positively stained product significantly increased by days 7 and 14 when compared with the level at day $2(\mathrm{P}<0.01)$, and no statistically significant difference was observed between days 7 and 14 $(\mathrm{P}>0.05)$. Peak expression occurred at day 7 and gradually decreased with time. The quantity of positive expression products significantly reduced to the level observed at day 2 by day 28 following the injection; a statistically significant difference was observed when compared with the levels at days 7 and $14(\mathrm{P}<0.01)$. There was no significant difference $(\mathrm{P}>0.05)$ when compared with the day 2 level.

For the sham-transfected and blank control groups, positive staining, indicative of IGF-1 expression, was only observed in glial cells at day 2 and was not detected in the motor neurons. However, the quantity of positively stained products in the motor neurons significantly increased at days 7 and 14 when compared with the level at day $2(\mathrm{P}<0.01)$; there was no significant difference between days 7 and 14 ( $\mathrm{P}>0.05)$. Peak expression occurred at day 7 and gradually decreased with time. The expression level at day 28 was significantly lower than the levels at days 7 and 14, and slightly lower than the level at day 2; statistically significant differences were observed when comparing the expression levels at days 2, 7 and 14.

Comparison between groups at each time point. IGF-1 expression levels in the hIGF-1 treatment group were higher compared with those in the other two groups and the difference was statistically significant $(\mathrm{P}<0.01)$. No significant difference in IGF-1 expression levels was identified between the sham-transfected and blank control groups $(\mathrm{P}>0.05$; Table I).

Detection of apoptotic motor neurons in the anterior horn of the spinal cord. TUNEL staining of apoptotic cell nuclei was brownish yellow, while normal cell nuclei were blue-violet. The apoptotic cell counts determined through TUNEL staining (Fig. 1) at different time points, revealed that the number of motor neuron cells undergoing apoptosis was at a maximum 7 days after sciatic nerve injury and gradually decreased by days 14 and 21 . The apoptotic cell count in the hIGF-1 treatment group was lower compared with those in the sham-transfected and blank control groups and the difference was statistically significant $(\mathrm{P}<0.01$; Table II). The apoptotic cells were motor neuron and glial cells.

Motor neuron cell count in the anterior horn of the spinal cord and pathological changes

Observations between time points for the groups. At day 2 following surgery, there was no evident difference in the number of motor neuron cells in the anterior horn of the spinal cord compared with the number in the normal spinal cord side. 
Table II. Number of motor neurons positive for TUNEL staining in the anterior horn of the spinal cord.

\begin{tabular}{llll}
\hline Group & Day 7 & Day 14 & Day 21 \\
\hline Blank control & $5.60 \pm 1.520$ & $4.6 \pm 1.14$ & $3.08 \pm 1.320$ \\
Sham-transfected & $6.20 \pm 1.480$ & $5.4 \pm 1.52$ & $2.72 \pm 1.400$ \\
hIGF-1 treatment & $2.40 \pm 0.551^{\mathrm{a}, \mathrm{b}}$ & $2.0 \pm 1.01^{\mathrm{a}, \mathrm{b}}$ & $1.68 \pm 0.891^{\mathrm{a}, \mathrm{b}}$ \\
\hline
\end{tabular}

Values are presented as the mean \pm standard deviation ( $\mathrm{n}=3$ per group per time point). ${ }^{\mathrm{a}} \mathrm{P}<0.01 \mathrm{vs}$. the blank control group; ${ }^{\text {b }} \mathrm{P}<0.01 \mathrm{vs}$. the sham-transfected group. TUNEL, terminal deoxynucleotidyl transferase-mediated dUTP nick end labeling; hIGF-1, human insulin growth factor-1.

Table III. Number of motor neuron cells in the anterior horn of the spinal cord.

\begin{tabular}{lcccc}
\hline Group & Day 2 & Day 7 & Day 14 & Day 28 \\
\hline Blank control & $20.80 \pm 2.390$ & $12.00 \pm 1.220$ & $9.20 \pm 1.300$ & $12.80 \pm 1.480$ \\
Sham-transfected & $20.00 \pm 2.550$ & $13.00 \pm 1.000$ & $12.40 \pm 1.140$ & $11.20 \pm 1.300$ \\
hIGF-1 treatment & $23.20 \pm 2.391$ & $16.20 \pm 1.301^{\mathrm{a}, \mathrm{b}}$ & $15.00 \pm 1.001^{\mathrm{a}, \mathrm{b}}$ & $17.20 \pm 1.921^{\mathrm{a}, \mathrm{b}}$ \\
\hline
\end{tabular}

Values are presented as the mean \pm standard deviation ( $\mathrm{n}=3$ per group per time point). ${ }^{\mathrm{a}} \mathrm{P}<0.01 \mathrm{vs.}$ the blank control group; ${ }^{b} \mathrm{P}<0.01 \mathrm{vs}$. the sham-transfected group. hIGF-1, human insulin growth factor-1.

Nissl bodies in the cytoplasm were clearly visible and showed coarse granular features. In addition, the nerve cells exhibited a little swelling and degeneration. Compared with the count at day 2, the number of motor neuron cells in the anterior horn gradually decreased between days 7 and 14 following surgery. In addition, Nissl body particles became thinner, smaller and even centrally dissolved. Motor neuron cells of the anterior horn showed deformation and neuronophagia, until ulceration disappeared after day 14. Compared with the count at day 14, the number of anterior horn cells in the spinal cord had increased by day 28 . The number of glial cells also increased, satellite phenomena appeared around the damaged motor neurons and new Nissl body particles were synthesized in the cytoplasm.

Comparison between groups at each time point. At day 2, there was no significant difference in the number of motor neurons among the three groups $(\mathrm{P}>0.05)$. The motor neuron cell numbers in the anterior horn of the spinal cord in the IGF-1 treatment group were increased at the day 7, 14 and 28 time points compared with those in the sham-transfected and control groups. In addition, in the IGF-1 treatment group the cytoplasmic Nissl bodies exhibited slightly reduced deformation than that in the other two groups and motor neuron repair occurred earlier. The effect on day 28 was particularly superior (Table III).

TEM observation. Neuropil changes were observed in the intramedullary section of the spinal cord by TEM at day 56 following surgery. In the hIGF-1 treatment group, the neuropil structure in the intramedullary section of the spinal cord was normal. However, in the sham-transfected group, the gap in the neuropil processus was large, the density of the axon mitochondria had increased and the myelin sheath of myelinated nerve fibers was loose. Vacuoles were observed in the neuropil of the blank control group.

\section{Discussion}

IGF-1 is a biological peptide with multiple functions, including neurotrophic and neural regulatory effects. The peptide is a member of the nerve growth factor family (16). Since Daughaday et al (17) identified this factor to be closely associated with a growth hormone $(\mathrm{GH})$, research into IGF-1 has developed. In addition, the emergence of recombinant IGF-1 biological products has developed a broader perspective of its use in the diagnosis and treatment of disease (18-20).

As neurotrophic factors have a short half-life, the application of genetic engineering is an effective method to provide a long-term supply of neurotrophic factors. In 1990, Wolff et al (21) first used a direct injection of DNA into muscles to transfer genes, for exogenous gene expression in skeletal muscle, which was a prelude to the use of plasmid vectors for gene therapy research. Reconnection of nerves following peripheral nerve injury is a necessary condition for the recovery of neurological function. The success of regeneration following peripheral nerve repair depends on the vitality of the central motor neurons and a suitable microenvironment for the renewal of growth. Substances such as nerve growth factors are important for the regeneration of the microenvironment. hIGF-1 has been confirmed to promote the regeneration of peripheral neurons. IGF-1 binds to the IGF-1 receptor on the surface of neurons at the site of injury, improves neuronal survival and promotes axonal regeneration and synapse formation, thereby contributing to the regeneration of the nerve (22).

Appropriately small gaps can use neural nutrition and chemotaxis to solve neural dislocation growth phenomena with more accurate docking. Certain studies $(23,24)$ have reported that $1-3 \mathrm{~mm}$ gaps achieve the best regenerative effect, which is why a $3-\mathrm{mm}$ gap was selected in the present study. A number of studies have used exogenous-promoting nerve growth factor 
to promote peripheral nerve regeneration during the repair of injury $(5,22,25)$. However, since the half-life of these factors is very short, the application of traditional methods requires repeated transfusions of these molecules and it is difficult to maintain effective concentrations in specific areas. The experimental application of genetic engineering involves using a plasmid as a carrier, which may extend the role of hIGF-1. In addition, compared with viral vectors, plasmid carriers are able to accommodate large fragments of DNA, which are transformed into circular DNA following transformation into target cells, and are not integrated and replicated. As a result, there is no viral infection or carcinogenic potential due to the presence of the viral vectors in the host $(26,27)$.

The results of the present study indicate that exogenous hIGF-1 administered in the area of the injured sciatic nerve can be transported to spinal motor neuron cells for expression, and that peak expression occurs at approximately day 7 . Apoptosis of motor neurons was observed at days 7, 14 and 21 following surgery. Apoptosis occurs following peripheral nerve injury, and may be the main route of neuronal cell death. The apoptosis peak occurred at approximately day 7 . The numbers of apoptotic cells in the hIGF-1 treatment group at the various time points were fewer than those in the sham-transfected and control groups, indicating that IGF-1 is able to reduce the apoptosis of motor neurons following peripheral nerve injury and protect motor neurons. At days 2 , 7, 14 and 28 following surgery, the number of motor neurons in the IGF-1 treatment group was higher compared with those in the other two groups. The degree of pathological change, including the central solution of Nissl bodies in neuron cells and neuronophagia, was lower in the IGF-1 treatment group than that of the other two groups. In addition, the phenomenon of oligodendroglia repairing motor neurons was more evident. All the results demonstrate that IGF-1 has the ability to protect motor neurons. Neuropil refers to the complex network area formed by the dendrites and axons of neurons and the neurites of gliocytes in the grey matter of the central nervous system, which are connected to each other. The neuropil is an important area for message exchange among central neurons; thus, injury, repair and regeneration functions of the spinal cord are accompanied by the degeneration and rebuilding of the neuropil. At day 56 following surgery, the ultrastructure of the neuropil in the IGF-1 treatment group was better than in the other two groups, indicating that the spinal cord was repaired more effectively.

The mechanism underlying hIGF-1 transfection into neural tissue cells and the detailed mechanism behind neuroprotection and nutrition remain unclear. Certain studies hypothesize that exogenous IGF-1 and -2 play a role in the central nervous system, primarily through targeted IGF protein-binding to neurons and glial cells (28). Nagano et al (29) hypothesized that the mechanism may involve the phosphoinositide 3-kinase signaling pathway. It has also been hypothesized (30) that the mechanism may function via the stimulating effects of trauma and other external factors causing increases in cell membrane permeability and the transport of exogenous plasmid DNA into the cell via a specific type of cell membrane transport, particularly pinocytosis. However, whether this hypothesis is correct requires further study.
The animal experiments in the present study demonstrate that IGF-1 administered in a liposome-mediated plasmid as carrier can be taken up by damaged axons and transported to the spinal neurons. The IGF-1 was found to play the greatest role in the spinal cord 7-14 days after transfection, with a neurotrophic effect at the biological level. Thus, this preliminary study on peripheral nerve injury and the pathological changes of spinal motor neurons, indicates that following peripheral nerve injury, the application of exogenous IGF-1 protected spinal motor neuron function. In future studies, transgenic IGF-1 and biomaterials for tissue engineering may be combined together to promote nerve regeneration following peripheral nerve injury, and potentially provide a new method for nerve generation.

\section{References}

1. Wang SR, Sun ZR, Dai YM, et al: Expression of ciliary neurotrophic factor of spinal cord motor neuron after sciatic nerve injury and changes after acupuncture intervention in rats. Zhongguo Zuzhi Gongcheng Yanjiu 8: 6954-6957, 2004 (In Chinese).

2. Bothwell M: Insulin and somatemedin MSA promote nerve growth factor-independent neurite formation by cultured chick dorsal root ganglionic sensory neurons. J Neurosci Res 8: 225-31, 1982.

3. Godińez-Gómez R, Trujillo-Hernández B, Tene CE, et al: Electrophysiological abnormalities in type 2 diabetic patients with reduced levels of insulin-like growth factor I. J Int Med Res 34: 21-29, 2006.

4. Zeng JZ, Dong KL, Li GC and Li LM: Effect of xiaokeling concentration fluid on mRNA expression of insulin-like growth factor-1 in sciatic nerve of Streptozotocin-induced diabetic rats. Zhong Nan Da Xue Xue Bao Yi Xue Ban 30: 49-52, 2005 (In Chinese).

5. Wang W, Teng Y, Zhang MQ, et al: Function of insulin receptor and insulin-like growth factor receptor in Alzheimer's disease. Sichuan Shengli Kexue Zazhi 35: 112-115, 2013.

6. Kiryakova S, Söhnchen J, Grosheva M, et al: Recovery of whisking function promoted by manual stimulation of the vibrissal muscles after facial nerve injury requires insulin-like growth factor 1 (IGF-1). Exp Neurol 222: 226-234, 2010.

7. Hu WL, Hu LQ, Li SW and Zheng XM: Reconstruction of erectile reflex circuit by autologous vein graft combined with use of insulin-like growth factor. Zhonghua Yi Xue Za Zhi 84: 1280-1282, 2004 (In Chinese).

8. Emel E, Ergün SS, Kotan D, et al: Effects of insulin-like growth factor-1 and platelet-rich plasma on sciatic nerve crush injury in a rat model. J Neurosurg 114: 522-528, 2011.

9. Vögelin E, Baker JM, Gates J, Dixit V, Constantinescu MA and Jones NF: Effects of local continuous release of brain derived neurotrophic factor (BDNF) on peripheral nerve regeneration in a rat model. Exp Neurol 199: 348-353, 2006.

10. Lin YC, Oh SJ and Marra KG: Synergistic lithium chloride and glial cell line-derived neurotrophic factor delivery for peripheral nerve repair in a rodent sciatic nerve injury model. Plast Reconstr Surg 132: 251e-262e, 2013.

11. Zhang J, Lineaweaver WC, Oswald T, Chen Z, Chen Z and Zhang F: Ciliary neurotrophic factor for acceleration of peripheral nerve regeneration: an experimental study. J Reconstr Microsurg 20: 323-327, 2004.

12. Brinton RD and Wang JM: Therapeutic potential of neurogenesis for prevention and recovery from Alzheimer's disease: allopregnanolone as a proof of concept neurogenic agent. Curr Alzheimer Res 3: 185-190, 2006.

13. Saito I, Oka Y and Odaka M: Promoting nerve regeneration through long gaps using a small nerve tissue graft. Surg Neurol 59: 148-55, 2003.

14. Zhang P, Zhang C, Kou Y, et al: The histological analysis of biological conduit sleeve bridging rhesus monkey nerve injury with small gap. Artif Cells Blood Substit Immobil Biotechnol 37: 101-104, 2009.

15. Jiang B, Zhang P and Jiang B: Advances in small gap sleeve bridging peripheral nerve injury. Artif Cells Blood Substit Immobil Biotechnol 38: 1-4, 2010. 
16. Zheng WH, Kar S, Doré S and Quirion R: Insulin-like growth factor-1 (IGF-1): a neuroprotective trophic factor acting via the Akt-kinase pathway. J Neural Transm Suppl 60: 261-272, 2000.

17. Daughaday WH, Hall K, Salmon WD, Van den Brande JL and Van Wyk JJ: On the nomenclature of the somatomedins and insulin-like growth factors. Endocrinology 121: 1911-1922, 1987.

18. Rosen CJ and Pollak M: Circulating IGF-I: New perspectives for a new century. Trends Endocrinol Metab 10: 136-141, 1999.

19. Twigg SM and Baxter RC: Insulin-like growth factor (IGF)-binding protein 5 forms an alternative ternary complex with IGFs and the acid-labile subunit. J Biol Chem 273: 6074-6079, 1998.

20. Holman SR and Baxter RC: Insulin-like growth factor binding protein-3: factors affecting binary and ternary complex formation. Growth Regul 6: 42-47, 1996.

21. Wolff JA, Malone RW, Williams P, et al: Direct gene transfer into mouse muscle in vivo. Science 247: 1465-1468, 1990.

22. Pu SF, Zhuang HX and Ishii DN: Differential spatio-temporal expression of the insulin-like growth factor genes in regenerating sciatic nerve. Brain Res Mol Brain Res 34: 18-28, 1995.

23. Butí M, Verdú E, Labrador RO, et al: Influence of physical parameters of nerve chambers on peripheral nerve regeneration and reinnervation. Exp Neurol 137: 26-33, 1996.
24. Kou YH, Yin XF, Zhang PX and Jiang BG: Small-gap bridging technology for peripheral nerve injury repair and the new sleeve material. Beijing Da Xue Xue Bao 43: 647-651, 2011 (In Chinese).

25. Zhang ZJ, Gao BQ, Liang CY, Liao ZG, Xu JG and Liu YF: Insulin-like growth factor in the regeneration of facial nerve following injury. Zhongguo Zhongxiyijiehe Er Bi Yanhou $\mathrm{Ke}$ Zazhi 12: 7-9, 2004 (In Chinese).

26. Gyula A, Shoushu J, Agnes J, et al: Direct gene transfer and expression into rat heart in vivo. New Biol 3: 71, 1991.

27. Jiao S, Williams P, Berg RK, et al: Direct gene transfer into nonhuman primate myofibers in vivo. Hum Gene Ther 3: 21-33, 1992.

28. Sizonenko SV, Sirimanne ES, Williams CE and Gluckman PD: Neuroprotective effects of the N-terminal tripeptide of IGF-1, glycine-proline-glutamate, in the immature rat brain after hypoxic-ischemic injury. Brain Res 922: 42-50, 2001.

29. Nagano T, Nakamura M, Nakata K, et al: Effects of substance $P$ and IGF-1 in corneal epithelial barrier function and wound healing in a rat model of neurotrophic keratopathy. Invest Ophthalmol Vis Sci 44: 3810-3815, 2003.

30. Zhang SQ, Liu L, Zhang JQ, et al: Expression of exogenous gene at the site of anastomoses of peripheral nerve. Tong Ji Yi Ke Da Xue Xue Bao 33: 455-458, 2004 (In Chinese). 